\title{
$q$-Calculus and the Symmetrized Radioactive Decay Law
}

\author{
Shawn K. Eastmond \\ Independent Researcher, Bridgetown, Barbados \\ shawn.eastmond@gmail.com
}

July 7,2021

\begin{abstract}
The radioactive decay law was first formulated by Ernest Rutherford and Frederick Soddy in 1902. As a well-known law, one of its primary applications is to determine the dates of ancient specimens. The process is known as radiocarbon dating and is subjected to the known properties of radioactive nuclei. In this paper, we implement quantum calculus to express the solution of the radioactive decay equation in symmetrized $q$-exponential form. Also, we explore a $q$-analog of the decay constant using Tsallis logarithmic function for various miscellaneous $q$-values. Furthermore, the factor-label method was applied to our analysis to show that the correct units remained intact under the application of quantum calculus. In conclusion, our work suggests that a variation of the $q$-parameter was akin to the production of a new isotope for all $q$ in $(0,1)$; the superadditive regime.
\end{abstract}

Keywords: $q$-calculus, radioactive decay, half-life, factor-label method

\section{Contents}

1 Introduction 2

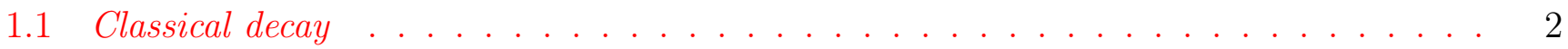

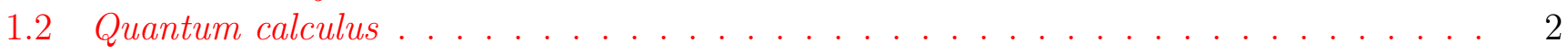

2 q-Basics 2

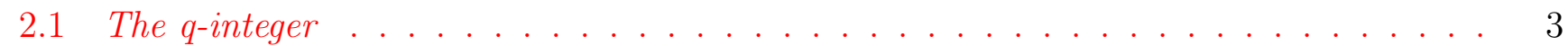

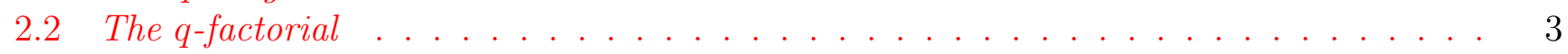

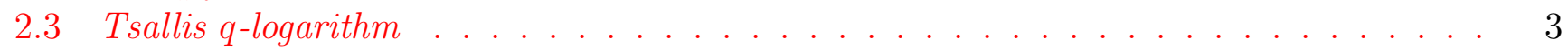

2.4 The q-derivative. . . . . . . . . . . . . . . . . . . . . . 3

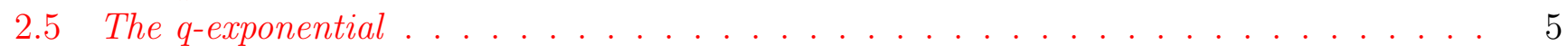

3 q-Exponential form $\quad 5$

3.1 The symmetrized form . . . . . . . . . . . . . . . . . . . 5 5

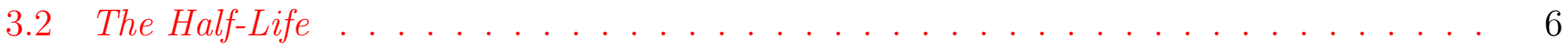

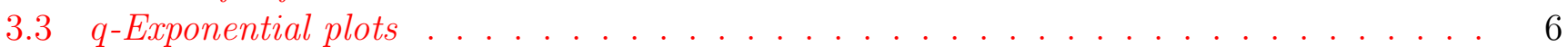

4 Factor-label method $\quad 7$

4.1 Unit-factor method . . . . . . . . . . . . . . . . . . . . 7 


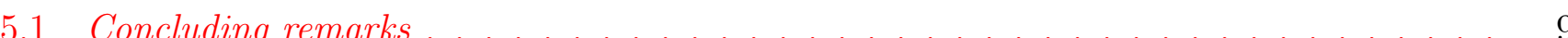

\section{Introduction}

In this paper, we will show by means of the power series method and quantum calculus in Section (3), that the radioactive decay law can be written in symmetrized quantum exponential form. In addition to that, we will use Tsallis nonextensive statistical mechanics to generate a number of superadditive curves. Superadditive curves are defined as having a $q$-parameter that is less than unity, i.e., in our case $q \in(0,1)$.

To generate the superadditive curves, we intend to exploit a special property of the $q$ logarithm based on Tsallis statistics. Our results will then be applied to the radiaactive decay constant. Finally, in Section (4) we will use the factor-label method to show that the units remained intact under a variation of the $q$-parameter.

\section{$1.1 \quad$ Classical decay}

A known solution to the radioactive decay equation can be yielded from an elementary technique in ordinary differential equations known as the seperation of variables[1]. Of course, this is not the only method to solve such an equation. Another method which ensures an identical solution to the previous equation is the power series method.

However, if we let the initial conditions of our system be as follows: at time $t=0, N(t)=N_{o}=$ $a_{o}$. Then the solution by the power series method is determined by substituting Eq.(1.2) in Eq.(1.1) to give Eq.(1.3),

$$
\begin{gathered}
\frac{d N}{d t}=-\lambda N \\
N(t)=\sum_{n=0}^{n=\infty} a_{n} t^{n} \\
\sum_{n=0}^{n=\infty}(n+1) a_{n+1} t^{n}+\lambda \sum_{n=0}^{n=\infty} a_{n} t^{n}=0 .
\end{gathered}
$$

The terms $a_{n}$ and $a_{n+1}$ are related and hence the power series solution in ref.[2] is attained by substituting $a_{n}$ in Eq.(1.2) where,

$$
a_{n}=\frac{(-1)^{n}(\lambda)^{n}}{n !} a_{0}
$$

and

$$
N(t)=\sum_{n=0}^{n=\infty} \frac{(-1 t)^{n}(\lambda)^{n}}{n !} a_{0} .
$$

A quicker and perhaps more straightforward method to find the solution to Eq.(1.1) is by seperating variables[3]. The terms $N_{0}, N(t), \lambda$, and $t$ are: (i) the number of nuclei at $t=0$, (ii) the number of nuclei at $t>0$, (iii) the radioactive decay constant, and (iv) the time $t$, respectively. Additionally, if we integrate Eq.(1.1) using the method of seperation of variables, we get

$$
\begin{aligned}
& \int_{N_{0}}^{N(t)} \frac{d N}{N}=-\lambda \int_{0}^{t} d t \\
& N(t)=N_{o} \exp (-\lambda t) .
\end{aligned}
$$

\section{$1.2 \quad$ Quantum calculus}

Quantum calculus in ref.[4] or sometimes called $q$-Calculus is calculus done without limits. The term $h$ in $q=e^{h}$ is used as a reminder of the planckian constant, while the term $q$ stands for "quantum". In the limit $h \rightarrow 0, q \rightarrow 1$ and the classical form of calculus is recovered[5]. Furthermore, another important concept in $q$-calculus which will be discussed later in more detail, is the derivative and its $q$-analog - it is important to point out that currently no $q$-analog for the generalized chain rule exist [5]. We will give an example of a function which satisfies the chain rule in Section (2), but bear in mind that several functions exist that do not satisfy this rule.

Nonetheless, for the sake of clarity the downward arrow between Eqs.(1.8) and (1.9) illustrates the removal of the limit. The limit in Eq.(1.8) is replaced by the number "1" and 
hence we get the well-known Jackson derivative in ref.[6-9] which is given by Eq.(1.9)

$$
\begin{gathered}
\frac{d f}{d x}=\lim _{q \rightarrow 1} \frac{f(q x)-f(x)}{q x-x} \\
\downarrow \\
\frac{d_{q} f}{d_{q} x}=\{1\} \frac{f(q x)-f(x)}{q x-x} . \\
2 \quad q \text {-Basics }
\end{gathered}
$$

\section{$2.1 \quad$ The q-integer}

Definition 2.1. Given that $0<q<1$ and $n \in \mathbb{N}$, the $q$-integer is defined by

$$
[n]_{q}= \begin{cases}\frac{1-q^{n}}{1-q}, & \text { if } q \neq 1 \\ n, & \text { if } q=1\end{cases}
$$

for $q \in \mathbb{R}$.

The next definition we will consider is the $q$ factorial in [10].

\section{$2.2 \quad$ The q-factorial}

Definition 2.2. Let $0<q<1$ and $n \in \mathbb{N}$, then the $q$-factorial is defined as

$$
\begin{aligned}
& {[n]_{q} !=} \\
& \begin{cases}{[n]_{q}[n-1]_{q} \ldots[1]_{q},} & n=1,2,3, \ldots \\
1, & n=0\end{cases}
\end{aligned}
$$

\subsection{Tsallis q-logarithm}

Definition 2.3. Let $q$ be a real parameter defined on the open interval $(0,1)$, then the Tsallis $q$-logarithm is defined as

$$
\begin{aligned}
& \ln _{q}(x)= \\
& \begin{cases}\ln (x), & x>0, \text { and } q=1 \\
\frac{x^{1-q}-1}{1-q}, & x>0, \text { and } q \neq 1 \\
\text { undefined, } & \text { if } x \leq 0\end{cases}
\end{aligned}
$$

Therefore, using Def.(2.3), we conveniently show that in the limit as $q \rightarrow 1, \ln _{q}(x)$ goes to

$$
\lim _{q \rightarrow 1}\left\{\ln _{q}(x)\right\}=(-1) \lim _{q \rightarrow 1} \frac{d}{d q}\left(x^{1-q}\right),
$$

and

$$
\lim _{q \rightarrow 1}\left\{\ln _{q}(x)\right\}=\lim _{q \rightarrow 1} \frac{d}{d u}\left(e^{u * \ln (x)}\right)=\ln (x),
$$

where we have made the substitution $(u=1-q)$, in Eq.(2.4).

Given Def.(2.3), our analysis of the radioactive decay law requires the use of Tsallis nonextensive statistical mechanics and probability theory. Therefore, we will give a brief description of how we will use the theory to justify our results. Now given two independent systems $Q^{\alpha}$ and $Q^{\gamma}$; thereby, from probability theory we know that $p\left(Q^{\alpha}+Q^{\gamma}\right)=p\left(Q^{\alpha}\right) p\left(Q^{\gamma}\right)$. It follows then that-Tsallis entropy- $S_{q}\left(Q^{\alpha}+\right.$ $\left.Q^{\gamma}\right)=S_{q}\left(Q^{\alpha}\right)+S_{q}\left(Q^{\gamma}\right)$, which is the limiting case - representing additivity when $q=1$-and satisfies Boltzmann-Gibbs statistics.

For non-integer representaions, i.e., $q \in$ $(0,1)$ we get the well-known Tsallis nonextensive statistical mechanics - extremization - and hence $S_{q}\left(Q^{\alpha}+Q^{\gamma}\right)=S_{q}\left(Q^{\alpha}\right)+S_{q}\left(Q^{\gamma}\right)-(q-$ 1) $S_{q}\left(Q^{\alpha}\right) S_{q}\left(Q^{\gamma}\right)$ in ref.[11]. Thus whenever the entropic parameter $q<1$-superadditivity-it must be true that $S_{q}\left(Q^{\alpha}+Q^{\gamma}\right) \geq S_{q}\left(Q^{\alpha}\right)$ $+S_{q}\left(Q^{\gamma}\right)$.

\subsection{The q-derivative}

Definition 2.4. Let $f(x)$ be a function that is differentiable and hence continuos; then its $q$-derivative is define as

$$
\left(D_{q} f\right)(x)=\frac{f(x)-f(q x)}{(1-q) x}, \quad x \neq 0,
$$

where $q$ in quantum calculus is described as the so-called $q$-parameter and varies strictly between 0 and 1 .

Clearly, from Def.(2.4), in the limit that $q \rightarrow 1$,

$$
\lim _{q \rightarrow 1}\left(D_{q} f\right)(x)=\frac{d f(x)}{d x}
$$


and we recover the classical form of the derivative. Likewise, following from the definition of the $q$-derivative[12], for constants $\alpha$ and $\beta$ there exist a linear relationship such that

$$
\begin{aligned}
D_{q}\{\alpha f(x)+ & \beta g(x)\} \\
& =\alpha D_{q}\{f(x)\}+\beta D_{q}\{g(x)\} .
\end{aligned}
$$

The next formula we shall consider is the product rule. In traditional-classical-calculus, if we take into consideration the product of two functions, $f(x) * g(x)$ - the derivative can be applied to the first function while holding the second function, followed by an addition sign and then the derivative is applied to second function while holding the first function [13]. Therefore, we will show that the product rule in $q$-calculus is nearly identical to its counterpart in classical calculus. The only difference between them is the $q$-parameter appears in the argument of the first function, i.e., $f(x)$ becomes $f(q x)$ and the $q$-derivative operates on each individual function as it would in classical calculus. Henceforth, using Eqs.(2.6) and (2.8), we may write the product formula for $x \neq 0$ as

$$
\begin{aligned}
& D_{q}\{f(x) g(x\})= \frac{f(q x) g(q x)-f(x) g(x)}{(q-1) x} \\
&= \frac{f(q x) g(q x)-f(q x) g(x)}{(q-1) x} \\
&+ \frac{f(q x) g(x)-f(x) g(x)}{(q-1) x} \\
&= \frac{f(q x)(g(q x)-g(x))}{(q-1) x} \\
&+\frac{(f(q x)-f(x)) g(x)}{(q-1) x} \\
&=f(q x) D_{q} g(x) \\
&+D_{q} f(x) g(x) .
\end{aligned}
$$

If we consider an interchange of the functions $f(x)$ and $g(x)$, then

$$
\begin{aligned}
D_{q}\{f(x) & g(x\}) \\
& =f(x) D_{q} g(x)+g(q x) D_{q} f(x) .
\end{aligned}
$$

It follows then that Eqs.(2.9 and 2.10) are equivalent forms. We say these forms are symmetric since an interchange of any of the two functions does not change the result. The symmetric nature of quantum calculus is important to some physicist because of its application to algebraic objects called quantum groups ${ }^{1}$ in [10]. A quantum group can be viewed as a Hopf algebra, i.e., a bialgebra - an algebra and a co-algebra - with an antipode. That is, for a Lie algebra $S$ :

- $S \times S \rightarrow S \quad$ (multiplicative map),

- $S \rightarrow S: g \rightarrow g^{-1} \quad$ (inversion map),

- $\Delta: S \rightarrow S \otimes S \quad$ (comultiplication),

- $\delta: S \rightarrow S$ (antipode), and

- $\zeta: S \rightarrow \mathbb{R} \quad$ (counit).

In like manner, the quotient rule and a nongeneralized example that leads to a limited definition of the chain rule-- no generalized chain rule currently exist - are given by[5],

$$
\begin{aligned}
D_{q}\left\{\frac{f(x)}{g(x)}\right\} & \\
= & \frac{1}{(q-1) x}\left\{\frac{f(q x)}{g(q x)}-\frac{f(x)}{g(q x)}\right\} \\
+ & \frac{1}{(q-1) x}\left\{\frac{f(x)}{g(q x)}-\frac{f(x)}{g(x)}\right\} \\
& =\frac{1}{g(q x)}\left\{\frac{f(q x)-f(x)}{(q-1) x}\right\} \\
+ & 1 \text { (q }-1) x\left\{\frac{f(x) g(x)-f(x) g(q x)}{g(q x) g(x)}\right\} \\
+ & \frac{1}{g(q x) g(x)}\left\{\frac{1}{g(q x)} D_{q} f(x)\right. \\
= & \frac{g(x) D_{q} f(x)-f(x) D_{q} g(x)}{g(q x) g(x)} .
\end{aligned}
$$

\footnotetext{
${ }^{1}$ Quantum groups are algebraic objects that are needed in the description of the $2+1$ gravity.
} 
And for some arbitrary function of the form $f(u(x))=f\left(\alpha x^{\beta}\right)$ in [5], where $\alpha$ and $\beta$ are constants, we get the following equation for a special case of the chain rule as depicted in ref.[14],

$$
\begin{array}{r}
D_{q}\{f(u(x))\}= \\
D_{q}\left\{f\left(\alpha x^{\beta}\right)\right\}=\frac{f\left(\alpha q^{\beta} x^{\beta}\right)-f\left(\alpha x^{\beta}\right)}{(q-1) x} \\
=\frac{f\left(\alpha q^{\beta} x^{\beta}\right)-f\left(\alpha x^{\beta}\right)}{\alpha q^{\beta} x^{\beta}-\alpha x^{\beta}} \times \frac{\alpha q^{\beta} x^{\beta}-\alpha x^{\beta}}{(q-1) x} \\
=\left(D_{q^{\beta}} f\right)(u(x)) D_{q}(u(x)) .
\end{array}
$$

\subsection{The q-exponential}

Definition 2.5. The $q$-exponential function(with $q \in(0,1))$ is defined by

$$
e_{q}(x)=\sum_{n=0}^{\infty} \frac{x^{n}}{[n]_{q} !} .
$$

It should be obvious - from Def.(2.2) - that the classical exponential function $\left(e^{x}\right)$ is recovered in the limit as $q \rightarrow 1$. The term in the denominator of Eq.(2.13); $[n]_{q} !=[1]_{q}[2]_{q} \ldots[n]_{q}$ in [15] is the only major difference between $e^{x}$ and $e_{q}(x)$ and this is where the symmetry and invariance arises from. If $q \rightarrow \frac{1}{q}$ and $[n]_{q}=\frac{q^{n}-q^{-n}}{q-q^{-1}}$, then $e_{q}(x)=e_{\frac{1}{q}}(x)$ are equivalent. Again, the fact that in the limiting case one can recover the classical form makes quantum calculus exceptionally beautiful[16].

\section{$3 \quad q$-Exponential form}

\subsection{The symmetrized form}

The precursory definition of the $q$-exponential function in Subsection (2.5) will enable us to write the solution for the radioactive decay equation by means of a suitable power series[17]. Conversely a $q$-exponential function based on Tsallis nonextensive statistical mechanics will be written in terms of Def.(2.3). That is, $\lambda$ will be written in terms of Tsallis $q$-logarithm. It will then be shown graphically - if we let the $q$-parameter vary for values strictly between 0 and 1 [18], that the classical form is indeed recovered whenever $q \rightarrow 1$.

We shall accomplish this by choosing a known isotope of oxygen - oxygen-22 - in [19] with a corresponding half-life of 2.25 seconds. The choice of isotope is completly random, and there exist no reason why any other isotope with a known half-life would not serve the same purpose. The modified decay constant is the given by

$$
\lambda_{q}=\frac{1}{2.25 s} \times \ln _{q}(2) .
$$

That being said, let the power series for $N(t)$ be defined as depicted in Eq.(1.2); then

$$
\frac{d_{q} N(t)}{d_{q} t}=\sum_{n=0}^{\infty}[n]_{q} a_{n} t^{n-1} .
$$

Now given that $[0]_{q}=0$, then

$$
\frac{d_{q} N(t)}{d_{q} t}=[0]_{q} a_{o} t^{-1}+\sum_{n=1}^{\infty}[n]_{q} a_{n} t^{n-1}
$$

for $n \geq 1$. We then substitute Eq.(3.3) in Eq.(1.1) and this gives,

$$
\sum_{n=0}^{\infty}[n+1]_{q} a_{n+1} t^{n}+\lambda \sum_{n=0}^{\infty} a_{n} t^{n}=0 .
$$

The terms in Eq.(3.4), which are of the form $a_{n}$ and $a_{n+1}$ [20] are the coefficients of $t^{n}$, therefore we must equate them, which gives

$$
a_{n+1}=-\frac{\lambda a_{n}}{[n+1]_{q}} .
$$

The relationship between $a_{n}$ and $a_{n+1}$ allows us to generate the following terms:

$$
\begin{gathered}
a_{1}=-\frac{\lambda a_{0}}{[1]_{q}} \\
a_{2}=\frac{(-\lambda)(-\lambda) a_{0}}{[1]_{q}[2]_{q}}, \\
a_{3}=\frac{(-\lambda)(-\lambda)(-\lambda) a_{0}}{[1]_{q}[2]_{q}[3]_{q}}, \\
a_{4}=\frac{(-\lambda)(-\lambda)(-\lambda)(-\lambda) a_{0}}{[1]_{q}[2]_{q}[3]_{q}[4]_{q}},
\end{gathered}
$$




$$
\begin{aligned}
& a_{5}=\frac{(-\lambda)(-\lambda)(-\lambda)(-\lambda)(-\lambda) a_{0}}{[1]_{q}[2]_{q}[3]_{q}[4]_{q}[5]_{q}}, \text { and } \\
& a_{6}=\frac{(-\lambda)(-\lambda)(-\lambda)(-\lambda)(-\lambda)(-\lambda) a_{0}}{[1]_{q}[2]_{q}[3]_{q}[4]_{q}[5]_{q}[6]_{q}} .
\end{aligned}
$$

Given that,

$$
[1]_{q}[2]_{q}[3]_{q} \ldots[n]_{q}=[n]_{q} !
$$

we may write $a_{n}$ as,

$$
a_{n}=\frac{(-1)^{n} \lambda^{n} a_{0}}{[n]_{q} !} .
$$

We may now substitute $a_{n}$ in Eq.(1.2), giving us

$$
N_{q}(t)=N_{0} \sum_{n=0}^{\infty} \frac{(-1)^{n} \lambda^{n} t^{n}}{[n]_{q} !}
$$

and

$$
N_{\frac{1}{q}}(t)=N_{q}(t)=N_{0} e_{q}(-\lambda t)=N_{0} e_{\frac{1}{q}}(-\lambda t)
$$

which is the q-analog of the symmetrized radioactive decay law. As a result of $e_{q}(x)=e_{\frac{1}{q}}(x)$, $N_{\frac{1}{q}}(t)=N_{q}(t)$ must be symmetric whenever $[n]_{q}=\frac{q^{n}-q^{-n}}{q-q^{-1}}$.

\subsection{The Half-Life}

The half-life $\left(t_{1 / 2}\right)$ is the time it takes for the number of radioactive nuclei to decrease by half the original value [21]. In the classical form, it is written as

$$
t_{1 / 2}=\frac{\ln (2)}{\lambda}
$$

where $\lambda$ is the radioactive constant. The fact that $\ln _{q}(x)$ exist for $x>0$ and $q \neq 1$, implies we may write $t_{1 / 2}$ as $t_{(1 / 2) O x y 22}=\frac{\ln _{q}(2)}{\lambda_{q}}$, where $t_{(1 / 2) O x y 22}$ is the half-life of oxygen-22. Again, we can move in the reverse direction to recover the classical form when $q \rightarrow 1$ as depicted below in Eq.(3.17),

$$
\begin{aligned}
t_{1 / 2} & =\lim _{q \rightarrow 1}\left\{t_{(1 / 2) \text { Oxy } 22}\right\} \\
& =-\lambda^{-1} \lim _{q \rightarrow 1} \frac{d}{d q}\left\{2^{1-q}\right\} \\
& =\frac{\ln (2)}{\lambda} .
\end{aligned}
$$

In addition to that, we will complete our plots with an equation of the form $\tilde{e}_{q}^{l n_{q}(x)}$, where tilde diferentiates $\tilde{e}_{q}^{l n_{q}(x)}$ from Def.(2.5) - thus we take advantage of a property of Tsallis functions, which is related to nonextensive statistical mechanics $^{2}$ - for several miscellaneous $q$-values. Tsallis functions are credited to Constantin Tsallis - 1988 - and have a variety of applications not only in physics but also in engineering and applied mathematics. Note that in [22],

$$
\ln _{q} e_{q}^{x}=e_{q}^{\ln x}=x, \quad \forall x
$$

where

$$
e_{q}^{x} \equiv[1+(1-q) x]^{\frac{1}{1-q}} .
$$

Remark 1. A function is said to be strictly increasing on an interval $I$, if $\forall p, q \in I, f(q)>$ $f(p)$. It turns out that the $q$-logarithm is a strictly increasing function. The action of taking the first derivative with respect to $x$, given Def.(2.3), when $q \neq 1$ and $x>0$, gives

$$
\frac{d}{d x} \ln _{q}(x)=\frac{1}{x^{q}}
$$

This result holds true $\forall q$.

\section{$3.3 \quad q-E x p o n e n t i a l$ plots}

In order to plot the superadditive curves, the miscellaneous $q$-values in Table (1) were attained from the open interval $(0,1)$; that is, $q \in(0,1)$. We selected $q$-values of: $0.1,0.2,0.3,0.7$, and 0.9 respectively. These values were substituted in the Tsallis $q$-logarithm- $\lambda_{q}$-and the numerical answers were divided by the half-life of oxygen-22 which is approximately 2.25 seconds.

\footnotetext{
2 Tsallis nonextensive statistical mechanics is a generalization of Boltzmann-Gibbs statistical mechanics.
} 
The final result is substituted into $\gamma_{0} N_{q}(t)$ and plotted for values of $t=[0,20]$, since $t_{(1 / 2) O x y 22}=\frac{\ln _{q}(2)}{\lambda_{q}}$. This will permit us to visualize the behavior of a curve; for some $q \in$ $(0,1)$. Nevertheless, in the limiting case (when $q \rightarrow 1$ ), we have made the assumption that the plots - the Tsallis q-exponetial plots - should regain their classical definition. On the other hand, as $q \rightarrow 0$, the curves should deviate away from the classical exponential definition.

Table 1: Miscellaneous quantum-values

\begin{tabular}{ccc}
\hline$q$ & $\frac{x^{1-q}-1}{1-q}\left\{\frac{x^{1-q}-1}{1-q}\right\} / t_{(1 / 2) \text { Oxy } 22}\left(s^{-1}\right)$ \\
\hline 0.1 & 0.962 & 0.428 \\
0.2 & 0.926 & 0.412 \\
0.3 & 0.892 & 0.396 \\
0.7 & 0.770 & 0.342 \\
0.9 & 0.718 & 0.319 \\
\hline
\end{tabular}

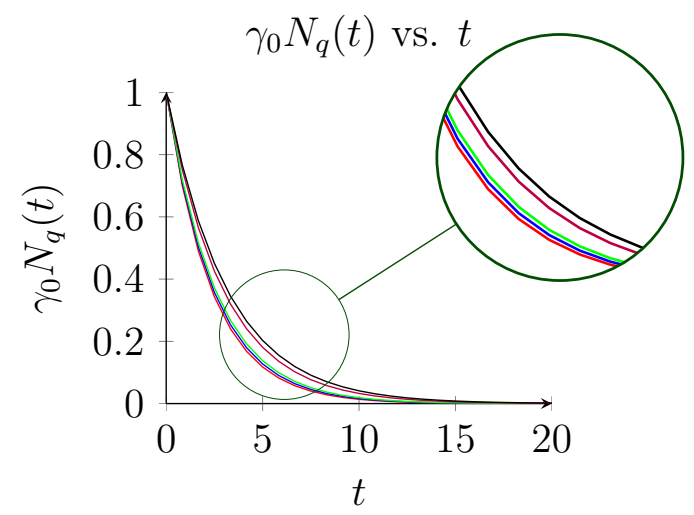

Figure 1: The $q$-exponential plots are based on the Tsallis $q$-logarithmic function. Using several miscellaneous $q$-values, in particular, 0.1, 0.2, 0.3, 0.7 , and 0.9 we plotted $\gamma_{0} N_{q}(t) v s$. $t$, where $\gamma_{0}$ is a constant.

The exponential plots in Fig.(1) illustrate a remarkably simple idea that is in alignment with the initial ansatz - the classical form should be recovered in the limiting case. As the $q$ parameter is varied; for instance, when $q=0.1$ (red) the exponential plot decayed faster than the other plots. On the contrary, for $q=0.9$ (black) the decay occurred at a slower rate. This suggests that as $q \rightarrow 0$ radioactive decay occurs faster. In words, faster decays correspond to more intense radiation[23], which was observed in our plots.

Finally, it is clear from our plots that a variation in the $q$-parameter (as $q \rightarrow 0$ ) has the effect of increasing the radioactive intensity. In contrast, as $q \rightarrow 1$ the classical form of the radioactive law is recovered. Therefore, the initial assumptions made with regards to Fig.(1) are justified and validated.

\section{Factor-label method}

\subsection{Unit-factor method}

In this Section, we will apply the unit-factor method in [24] to the modified radioactive decay laws in Subsections (3.1) and (3.2), and their classical counterpart. The objective here is to confirm whether or not the units of these equations are invariant under a variation of the $q$ parameter. The classical form of the radioactive law is given by:

$$
N(t)=N_{0} \exp (-\lambda t),
$$

and has units of

$$
1=\text { nucter }=\text { nucter } * U .
$$

The quantum form of the radioactive decay law-based on Tsallis statistics - is given by:

$$
\gamma_{0} N_{q}(t)=\tilde{e_{q}}(-\lambda t)
$$

and has units of

$$
1=\text { nucter }=\text { nucter } * U .
$$

And lastly, the quantum form of the radioactive decay law - based on Def.(2.5) - is given by:

$$
N_{\frac{1}{q}}(t)=N_{q}(t)=N_{0} e_{q}(-\lambda t)=N_{0} e_{\frac{1}{q}}(-\lambda t)
$$

and has units of

$$
1=\text { sucter }=\text { nucter }=\text { nucter } * U \text {. }
$$

To justify these results, we take a closer look at the exponents. Therefore, for

$$
\lambda t=t * \frac{\ln (2)}{t_{(1 / 2)}},
$$


in Eqs.(4.1) and (4.5); the units are

$$
1 *(\text { seconds })^{-1} * \operatorname{seconds}=U .
$$

Here, $U$ is used in commemoration of the dimensionless unit uno. Now in terms of the radioactive decay constant, the units for the Tsallis function also remained intact. Thus we have

$$
\lambda_{q} t=\frac{\ln _{q}(2) * t}{t_{(1 / 2) O x y 22}}
$$

with units of,

$$
1 * \underline{(\operatorname{second})^{-1}} * \operatorname{seconds}=U
$$

Hence, Eqs.(4.8) and (4.10) have identical units. This stems from the fact that the quantumfactorial- $[n]_{q}$ ! - in Def.(2.5) has units of dimension unity.

\section{Conclusion}

Up until now, we had not discussed a rationale for the use of Tsallis q-logarithm in our analysis. Nevertheless, we were able to use Tsallis $q$-logarithm along with the property of its inverse function in this work because the solution of the radioactive decay equation has a $q$-exponential mathematical structure - a growth equation is also applicable here as well. Therefore, we plotted and visualized the behavior of a set of miscellaneous $q$-values, in the superadditive - superextensive - regime.

In Subsection (3.2) we briefly mentioned Tsallis nonextensive statistical mechanics which was developed on the general principles of Boltzmann-Gibbs (BG) statistical mechanics. The term nonextensive as applied here to nonextensive statistical mechanics arises from the fact that independent systems - for example $Q^{\alpha}$ and $Q^{\gamma}$-are not additive when $q \neq 1[25]$. In other words, the probability of two independent systems $Q^{\alpha}$ and $Q^{\gamma}$, for the entropic parameter $q \neq 1$ are nonadditive. Hence, the name nonextensive - nonadditive - statistical mechanics is given to the theory. The entropic parameter $q$ is a measure of the nonadditivity in the system[26].
Within the framework of Nonadditive statistical mechanics the Boltzmann-Gibbs entropy $S_{G B}$ is extremized and in the limit as the entropic parameter $q \longrightarrow 1$, the ordinary Boltzmann-Gibbs entropy is recovered. The entropic parameter $q$, is determined by the microscopic dynamics of the system and is characterized by the degree of nonadditivity. In addition, if $Q^{\alpha}$ and $Q^{\gamma}$ are two independent systems then from probability theory it must be true that $p\left(Q^{\alpha}+Q^{\gamma}\right)=p\left(Q^{\alpha}\right) p\left(Q^{\gamma}\right)$. But entropy is nonnegative so $S_{q} \geq 0$-Tsallis entropy - and hence, for $q<1$ we have the superadditivity condition or superextensitivity[26]. For $q=1$, we have additivity or extensitivity, and finally for $q>1$ we have subadditivity or subextensitivity.

In spite of what was previously mentioned about $S_{q}$, we shall elucidate here a bit more on the Tsallis entropy to reinforce the idea of nonadditivity; that is, when $q \neq 1$. Moreover, we will give a short but concrete mathematical description of the intimate connection between $S_{q}$ and Jackson's derivative; that does not appear obvious in the initial stages of this work. Therefore, Tsallis entropy for $Q^{\alpha}$ and $Q^{\gamma}$ is given by

$$
\begin{gathered}
S_{q}\left(Q^{\alpha}\right)=\frac{1-\sum_{i} p_{Q^{\alpha}}^{q}}{q-1}, \text { and } \\
S_{q}\left(Q^{\gamma}\right)=\frac{1-\sum_{j} p_{Q^{\gamma}}^{q}}{q-1} .
\end{gathered}
$$

respectively [27]. This means that,

$$
\begin{gathered}
S_{q}\left(Q^{\alpha}+Q^{\gamma}\right)=\frac{1-\sum_{i} \sum_{j} p_{Q^{\alpha}}^{q} p_{Q^{\gamma}}^{q}}{q-1} \\
=\frac{2-\sum_{i} p_{Q^{\alpha}}^{q}-\sum_{j} p_{Q^{\gamma}}^{q}}{q-1} \\
-\frac{\left(1-\sum_{i} p_{Q^{\alpha}}^{q}\right)\left(1-\sum_{i} p_{Q^{\gamma}}^{q}\right)}{q-1} \\
=\frac{1-\sum_{i} p_{Q^{\alpha}}^{q}+\frac{1-\sum_{i} p_{Q^{\gamma}}^{q}}{q-1}}{q-1} \\
-(q-1) \frac{\left(1-\sum_{i} p_{Q^{\alpha}}^{q}\right)}{q-1} \frac{\left(1-\sum_{i} p_{Q^{\gamma}}^{q}\right)}{q-1} \\
=S_{q}\left(Q^{\alpha}\right)+S_{q}\left(Q^{\gamma}\right)-(q-1) S_{q}\left(Q^{\alpha}\right) S_{q}\left(Q^{\gamma}\right) .
\end{gathered}
$$

The inspiration behind the development of Tsallis entropy $\left(S_{q}\right)$ is based upon the theory 
of multifractal geometry - non-integer representations of fractal dimensions. Nevertheless, Tsallis was able to put forward a concrete generalization of Claude Shannon's entropy; and later it was S. Abe in [28] who noticed a connection between Jackson's derivative and Tsallis entopy $\left(S_{q}\right)$ in nonextensive statistical mechanics.

To show that there is a connection between nonextensive statistical mechanics and the Jackson derivative we begin with the BG theory. Therefore, the BG entropy in discrete form is given by,

$$
S_{B G}=-\sum_{i} p_{i} \ln p_{i}
$$

Alternatively, the BG entropy in [29] can also be expressed as a continuous function which is given by

$$
S_{B G}=-\int d x p(x) \ln p(x) .
$$

However, this link or connection can be deduced by considering Tsallis generalization of the Shannon entropy in [30] which is given by

$$
S_{q}=\frac{1}{q-1}\left(1-\sum_{i} p_{i}^{q}\right) \text {. }
$$

On the other hand, the continuous form of the Tsallis entropy is given by

$$
S_{q}=\frac{1-\int d x[p(x)]^{q}}{q-1} .
$$

Hence, if we apply the definition of the Jackson derivative to the second term in parenthesis of Eq.(5.6), we get

$$
D_{q} \sum_{i} p_{i}^{x}=\frac{\sum_{i} p_{i}^{q x}-\sum_{i} p_{i}^{x}}{x(q-1)}
$$

In the limiting case when $x \rightarrow 1$ we have

$$
\lim _{x \rightarrow 1} D_{q} \sum_{i} p_{i}^{x}=\lim _{x \rightarrow 1} \frac{\sum_{i} p_{i}^{q x}-\sum_{i} p_{i}^{x}}{x(q-1)}=-S_{q}
$$

unmasking the connection that Abe noticed between $S_{q}$ and $D_{q}$.

\section{$5.1 \quad$ Concluding remarks}

We have shown in our analysis, that by use of the power series method and $q$-Calculus, it is possible to write a $q$-analog for the radioactive decay law. Additionally, we extended our analysis and showed that the radioactive decay constant can be written in terms of a $q$-analog-based on Tsallis statistics - while the units remained invariant in the limit as $q \rightarrow 1$. Furthermore, using Tsallis $q$-logarithmic function and the radioactive decay constant, we generated some superextensive plots. A methodical analysis of the plots - Fig.(1) - found that as $q \rightarrow 0$, the decay process occurred at a faster rate. In other words, the radioactive decay intensity increased.

On the contrary, as $q \rightarrow 1$ the plots regained the classical form of the radioactive decay law. Now for the modified half-life, $t_{(1 / 2) O x y 22}=2.25$ seconds and $\lambda_{q}=\left[\left(x^{1-q}-1\right) /(1-q)\right] / t_{(1 / 2) O x y 22}$, a variation in the $q$-parameter was akin to the production of a new isotope. This is synonymous with a phenomenon in nuclear physics known as isotopic transmutation - each isotope has its own characteristic radioactive decay constant. Note that while the half-life of the isotope - oxygen22 -remained constant, $\ln _{q}(2)$ changed $\forall q \in$ $(0,1)$.

In conclusion, we will extend our analysis in Sections (3) and (4) to systems with more than one radioactive decay chain. In particular, we will consider Bateman system of differential equations for stable daughter nuclide - two nuclide in a chain - with $\lambda^{d}=0, N^{d}(0)=0$, and $N^{d}(t)=N^{p}(0)\left(1-\exp \left(-\lambda^{p} t\right)\right)$. Here, $N^{p}(0)$ and $N^{d}(t)$ are the parent and daughter nuclide, respectively.

\section{References}

[1] W. T. Ang and Y. S. Park, Ordinary Differential Equations (Universal Publishers, 2008).

[2] A. Fitouhi and K. Brahim, Journal of Nonlinear Mathematical Physics 14, 324 (2007).

[3] A. King, S. Billingham, and S. R. Otto, 
Differential Equations (Cambridge University Press, 2003).

[4] M. Bohner and T. Hudson, International Journal of Applied Mathematics and Statistics 9, 19 (2007).

[5] V. Kac and P. Cheung, Quantum Calculus (Springer New York, 2012).

[6] A. Nemri and B. Selmi, Journal of Mathematical Analysis and Applications 359, 588 (2009).

[7] A. Nemri and B. Selmi, Indagationes Mathematicae 24, 491 (2013).

[8] K. Piejko, J. Sokól, and K. TrąbkaWięcław, Iranian Journal of Science and Technology, Transaction A: Science 43, 2879 (2019).

[9] A. Fitouhi and N. Bettaibi, Journal of Nonlinear Mathematical Physics 13, 492 (2006).

[10] T. Ernst, Journal of Nonlinear Mathematical Physics 10, 487 (2003).

[11] S. Abe, Physics Letters, Section A: General, Atomic and Solid State Physics 271 (2000), 10.1016/S0375-9601(00)00337-6.

[12] A. Lavagno and G. Gervino, Journal of Physics: Conference Series 174 (2009), 10.1088/1742-6596/174/1/012071.

[13] R. T. Smith and M. B. Martin, Calculus (McGraw-Hill Companies,Incorporated, 2007).

[14] J. Tariboon and S. K. Ntouyas, Advances in Difference Equations 16, 1 (2013).

[15] A. Fitouhi, K. Brahim, and N. Bettaibi, Journal of Nonlinear Mathematical Physics $12,586(2005)$.

[16] G. Yener and I. Emiroglu, Discrete and Continuous Dynamical Systems - Series S 8, 1435 (2015).
[17] D. Betounes, Differential Equations: Theory and Applications (Springer New York, 2010).

[18] T. Z. Mirković, S. B. Tričković, and M. S. Stanković, Journal of Inequalities and Applications 1 (2018), 10.1186/s13660-018-1928$\mathrm{Z}$.

[19] A. Brown, International Journal of Modern Physics E 26 (2017), $10.1142 /$ S0218301317400031.

[20] S. Fujita and S. V. Godoy, Mathematical Physics (Wiley, 2010).

[21] B. R. Martin, Nuclear and Particle Physics: An Introduction (Wiley, 2019).

[22] T. Yamano, Physica A: Statistical Mechanics and its Applications 305 (2002), 10.1016/S0378-4371(01)00567-2.

[23] K. Hentschel, F. Weinert, and D. Greenberger, Compendium of Quantum Physics (Springer Berlin Heidelberg, 2009).

[24] R. G. Mortimer, Mathematics for Physical Chemistry (Elsevier Science, 2013).

[25] Y. Okamoto, Nonextensive Statistical Mechanics and Its Applications (Springer Berlin Heidelberg, 2008).

[26] C. Tsallis, Introduction to Nonextensive Statistical Mechanics (Springer New York).

[27] M. Lyra and C. Tsallis, Physical Review Letters 80 (1998), 10.1103/PhysRevLett.80.53.

[28] S. Abe, Physics Letters, Section A: General, Atomic and Solid State Physics 244 (1997), 10.1016/S0375-9601(96)00832-8.

[29] T. Constantino and B. Edgardo, Continuum Mechanics and Thermodynamics 16 (2004), 10.1007/s00161-004-0174-4.

[30] R. J. V. Dos Santos, Journal of Mathematical Physics 38 (1997), 10.1063/1.532107. 